\title{
Crystal structure of $\Delta^{9}$ stearoyl-acyl carrier protein desaturase from castor seed and its relationship to other di-iron proteins
}

\section{Ylva Lindqvist ${ }^{1}$, Weijun Huang, Gunter Schneider and John Shanklin²}

\author{
Division of Structural Biology, Department of Medical Biochemistry \\ and Biophysics, Karolinska Institute, Doktorsringen 4, \\ S-171 77 Stockholm, Sweden and ${ }^{2}$ Department of Biology, \\ Brookhaven National Laboratory, Upton, NY 11973, USA \\ ${ }^{1}$ Corresponding author
}

The three-dimensional structure of recombinant homodimeric $\Delta^{9}$ stearoyl-acyl carrier protein desaturase, the archetype of the soluble plant fatty acid desaturases that convert saturated to unsaturated fatty acids, has been determined by protein crystallographic methods to a resolution of $2.4 \AA$. The structure was solved by a combination of single isomorphous replacement, anomalous contribution from the iron atoms to the native diffraction data and 6-fold non-crystallographic symmetry averaging. The 363 amino acid monomer consists of a single domain of $11 \alpha$-helices. Nine of these form an antiparallel helix bundle. The enzyme subunit contains a di-iron centre, with ligands from four of the $\alpha$-helices in the helix bundle. The iron ions are bound in a highly symmetric environment, with one of the irons forming interactions with the side chains of E196 and H232 and the second iron with the side chains of E105 and H146. Two additional glutamic acid side chains, from E143 and E229, are within coordination distance to both iron ions. A water molecule is found within the second coordination sphere from the iron atoms. The lack of electron density corresponding to a $\mu$-oxo bridge, and the long $(4.2 \AA)$ distance between the iron ions suggests that this probably represents the diferrous form of the enzyme. A deep channel which probably binds the fatty acid extends from the surface into the interior of the enzyme. Modelling of the substrate, stearic acid, into this channel places the $\Delta^{9}$ carbon atom in the vicinity of one of the iron ions.

Keywords: di-iron centre/electron transfer/fatty acid biosynthesis/protein crystallography

\section{Introduction}

Fatty acid biosynthesis in higher plants has attracted increased interest recently because of the possible use of plant oils as renewable sources for reduced carbon (Somerville and Browse, 1991; Ohlrogge, 1994). In plants, fatty acid biosynthesis occurs in the chloroplasts of green tissue or in the plastids of non-photosynthetic tissues (Harwood, 1988; Ohlrogge and Browse, 1995). The primary products in most plants are acyl carrier protein (ACP) esters of the saturated palmitic and stearic acids.

$\Delta^{9}$ Stearoyl-acyl carrier protein desaturase $\left(\Delta^{9}\right.$ desatur- ase) is a plastid-localized non-membrane-bound soluble desaturase that introduces the first double bond into saturated fatty acids, resulting in the corresponding monounsaturated fatty acids (Nagai and Bloch, 1966). Recently, several related soluble desaturases have been identified in the seed tissues of various plants that possess fatty acids with unusual double bond positions (Cahoon et al., 1992, 1994a,b). Members of this class of soluble desaturases are specific for a particular substrate chain length and introduce the double bond between specific carbon atoms by counting from the carboxyl end of the fatty acid, e.g. the $\Delta^{9}$ desaturase is specific for stearoyl-ACP, and introduces a double bond between carbon 9 and 10 (McKeon and Stumpf, 1982; Gibson, 1993). Initial desaturation reactions in animals and fungi, and subsequent desaturation reactions in plants, are mediated by a distinct class of fatty acid desaturases that are integral membrane proteins (Thiede et al., 1986; Stukey et al., 1990; Ohlrogge and Browse, 1995). Since most plants lack other desaturases that act on the 18:0 level, the ratio of saturated to unsaturated fatty acids in higher plants is controlled mainly by enzymes which catalyse the conversion of saturated to mono-unsaturated fatty acids.

$\Delta^{9}$ Desaturase cDNA encodes precursor proteins containing an N-terminal transit peptide for targeting to the plastid. For safflower and castor, the 33 residue transit peptide is cleaved off to yield a 363 amino acid mature desaturase polypeptide with an apparent mol. wt of $37 \mathrm{kDa}$ per subunit by SDS-PAGE (Shanklin and Somerville, 1991; Thompson et al., 1991; Fox et al., 1993). The enzyme occurs as dimers of $\sim 70 \mathrm{kDa}$ (McKeon and Stumpf, 1982; Shanklin and Somerville, 1991; Thompson et al., 1991). The enzymatic reaction requires molecular oxygen, $\mathrm{NAD}(\mathrm{P}) \mathrm{H}, \mathrm{NAD}(\mathrm{P}) \mathrm{H}$ ferredoxin oxido-reductase and ferredoxin (Nagai and Bloch, 1968).

Previous studies have shown that both soluble and membrane-bound $\Delta^{9}$ desaturases require non-haem iron for catalytic activity (Nagai and Bloch, 1968; Strittmatter et al., 1974). More recently, spectroscopic analysis and amino acid sequence comparisons (Fox et al., 1993, 1994) have established that the $\Delta^{9}$ desaturase contains a di-iron cluster. This class of di-iron proteins is characterized by two occurrences of the sequence motif $\mathrm{E}-\mathrm{X}-\mathrm{X}-\mathrm{H}$, spaced by $\sim 100$ amino acids, and includes the R2 subunit of ribonucleotide reductase and a methane monooxygenase (MMO) hydroxylase component (Nordlund et al., 1992; Fox et al., 1993).

We have initiated crystallographic studies of $\Delta^{9}$ desaturase as a first step towards elucidation of the catalytic mechanism of this enzyme (Schneider et al., 1992). Here we describe the three-dimensional structure of $\Delta^{9}$ desaturase and its relationship to that of other di-iron cluster-containing proteins. We also correlate the structural features of the iron centre with available biochemical and spectroscopic data. 

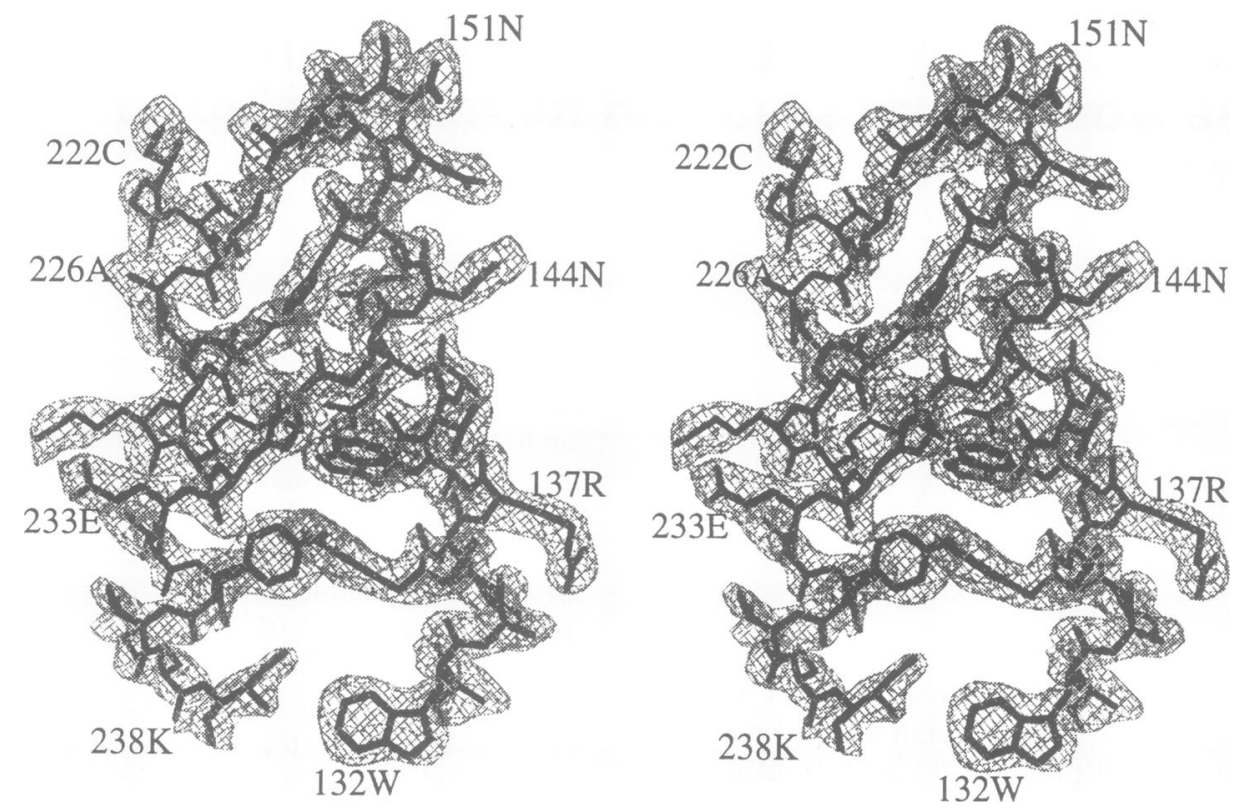

Fig. 1. Parts of the final, refined, averaged $\left.2\left|F_{0}\right|-\mid F_{\mathrm{c}}\right]$ electron density map at $2.4 \AA$ Resolution for $\Delta^{9}$ desaturase, contoured at $1.0 \sigma$. The refined model of $\Delta^{9}$ desaturase is superposed.

\section{Results and discussion}

\section{Electron density map and quality of the model}

The 6-fold averaged electron density for the main chain and side chains for most of the polypeptide chain is well defined (Figure 1). Exceptions are the first 18 residues at the $\mathrm{N}$-terminus, which are not defined in electron density and might be flexible in the crystal lattice. Residues 336347 , located in a loop region, are very poorly defined in the electron density maps and it is also in this part of the protein structure where the largest deviations from the non-crystallographic symmetry are found. The overall residue by residue real space correlation (Brändén and Jones 1990; Jones et al., 1991) between the model for one subunit and the 6-fold averaged $2 F_{0}-F_{c}$ electron density map is 0.76 . Criteria such as crystallographic $R$ factor $\left(R=22.0 \%, R_{\text {free }}=28.5 \%\right.$ with non-crystallographic symmetry restraints), good stereochemistry of the model (bond length r.m.s. of $0.008 \AA$ ), Ramachandran plot (only one outlier from the allowed regions per subunit, except glycine residues) and the observed hydrogen bonding pattern all indicate that the chain tracing for fatty acid desaturase is correct. There is very clear density for the peptide oxygen of Lys262, the residue with a disallowed main chain conformation.

The high average $B$-factor suggests that the molecule is flexible. The most ordered parts of the molecule are areas involved in dimer and hexamer interactions, whereas surface loops often have very high $B$-factors.

The major binding sites for the $\mathrm{Au}(\mathrm{CN})_{2}{ }^{-}$ions in the derivatized desaturase crystals are found close to the side chains of K56 and C61 at the surface of the molecule. One of the minor sites is internal, between the side chains of $\mathrm{H} 203$ and $\mathrm{C} 222$, and the second minor site is in the area where the $\mathrm{N}$-terminus of the chain probably is situated.

\section{Overall structure of the subunit}

The overall shape of the $\Delta^{9}$ desaturase subunit is a compact cylinder of dimensions $35 \AA \times 35 \AA \times 50 \AA$ with an accessible surface area of $16773 \AA^{2}$. Besides a $\beta$-hairpin loop at the very $C$-terminus of the chain, the subunit is composed mainly of helical secondary structures (see Figure 2) folded into one large domain. Nine of the total $11 \alpha$-helices form an antiparallel helical bundle with the topology shown in Figure 2A and in the schematic diagram of the subunit in Figure 2B.

The N-terminal part of the chain is disordered; no electron density is observed for the first 18 residues. The next 15 residues lack secondary structure and form an extended chain packing along the helix bundle with few specific interactions to stabilize its structure. The first helix, $\alpha 1$, composed of 23 residues, starts and ends in $3_{10}$ conformation and is very bent so that its first half forms a cap at one end of the bundle and its second part is the first helix of the bundle. The chain continues in the same direction forming hydrogen-bonded turns and a $3_{10}$ helix. The cap at the other end of the bundle is formed by helices $\alpha 2$ and $\alpha 9$ and the C-terminal $\beta$-hairpin. Four of these helices, $\alpha 3, \alpha 4, \alpha 6$ and $\alpha 7$, which are very long, $28,29,30$ and 31 residues respectively, contribute ligands to the di-iron centre.

Although $\alpha 3$ has a break in the helical structure in the middle at residue 107-108, $\alpha 3$ and $\alpha 4$ are symmetrical to $\alpha 6$ and $\alpha 7$ and can be superimposed with an r.m.s deviation of $1.39 \AA$ for 44 residues. Such a superposition also aligns the iron atoms to within $1.0 \AA$. The corresponding sequence alignments show that there is little sequence conservation besides the residues involved in binding the iron cluster (Figure 3). This superposition also orients the cap part of $\alpha 1$ on to $\alpha 2$. The connections between these helices also approximately superimpose although there is no detailed structural similarity.

Between $\alpha 3 b$ and $\alpha 4$ there is a protruding loop structure stabilized by several hydrogen-bonded turns. Helices $\alpha 5$, $\alpha 8, \alpha 10$ and $\alpha 11$, which is very curved, complete the bundle.

There are a large number of salt bridges, 25 , excluding 


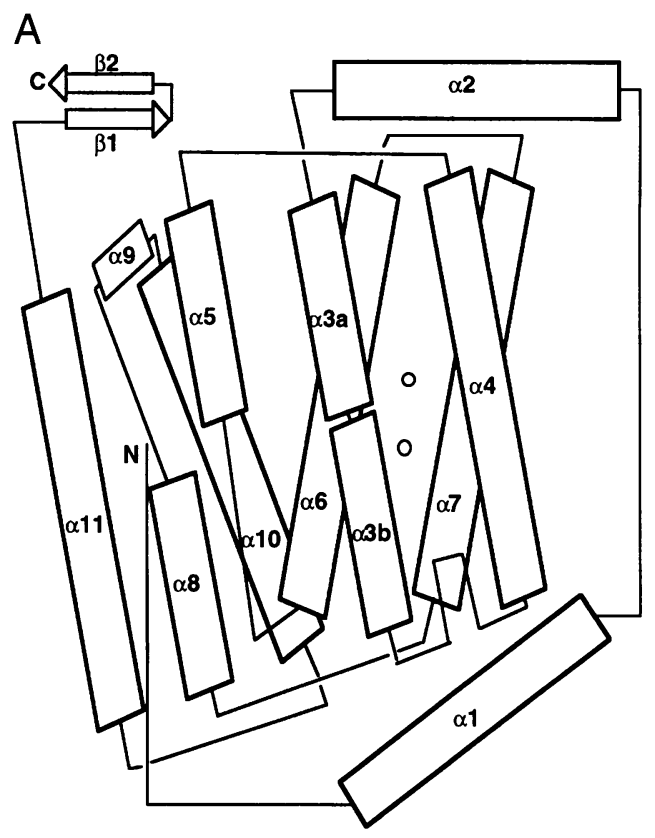

B

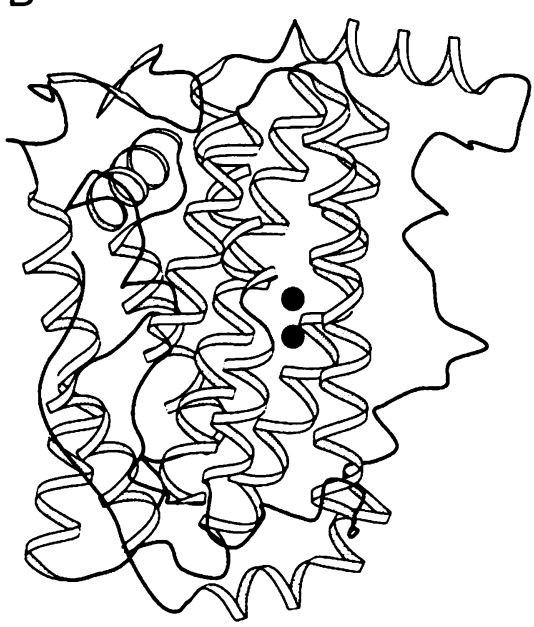

those interacting with the iron ions, within the subunit (Table I). This corresponds to 0.069 ion pairs per residue, higher than the average number of ion pairs per residue, 0.04 , derived from a survey of 38 high resolution protein structures (Barlow and Thornton, 1983). Seven of the salt bridges in $\Delta^{9}$ desaturase are involved in inter-helix interactions within the bundle, securing the correct mutual packing and, in some instances, correct orientation of the iron ligands. Eight pairs make intra-helix contacts, and three of the remaining are involved in anchoring the turns between bundle helices. Three pairs are involved in contacts to the $3_{10}$ helix and the loop after $\alpha 8$.

A peculiar feature of the $\Delta^{9}$ desaturase subunit is a rather flat surface formed by helices $\alpha 1, \alpha 6, \alpha 7, \alpha 10$ and $\alpha 11$. This surface is not involved in subunit-subunit contacts in the dimer but is accessible from the solution.

\section{The dimer}

The subunit-subunit interface in the dimer buries a surface area of $5826 \AA^{2}$ per dimer, $17.4 \%$ of the dimer area. These 2-fold interactions include extensive contacts
Fig. 2. (A) Topology diagram for the subunit of $\Delta^{9}$ desaturase. The $\alpha$-helices are labelled $\alpha 1-\alpha 11$. Limits of secondary structure elements are: $\alpha 1,36-51 ; \alpha 2,75-88 ; \alpha 3 \mathrm{a}, 91-106 ; \alpha 3 \mathrm{~b}, 108-118 ; \alpha 4,130-158$; $\alpha 5,161-176 ; \alpha 6,184-213 ; \alpha 7,215-245, \alpha 8,249-262 ; \alpha 9,278-290$; $\alpha 10,293-309 ; \alpha 11,317-341 ; \beta 1,349-353 ; \beta 2,359-363$. (B) Stereo view of the subunit of $\Delta^{9}$ desaturase. The location of the di-iron centre is indicated by black spheres. The figure was generated with the program Molscript (Kraulis, 1991).

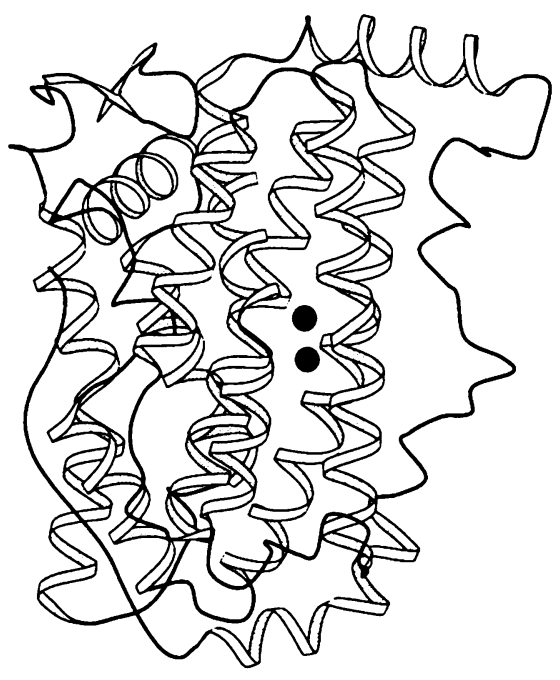

between helices in the bundles; from $\alpha 3 b$ to the same helix in the second subunit, between $\alpha 4$ and $\alpha 5$, and over $\alpha 2$ and $\alpha 4$ to the corresponding helices in the other subunit (see Figure 4). There are also many contacts between the protruding loop between $\alpha 3 b$ and $\alpha 4$ and the $\mathrm{N}$-terminal, $\alpha 3 b$, and $\alpha 5$. In addition, residues in the connection between $\alpha 1$ and $\alpha 2$ make contacts to $\alpha 4$ and $\alpha 5$ in the second subunit. There are three charged interactions in the dimer contact area, two of these involve residues from $\alpha 5$. The di-iron centres are separated by $>23 \AA$ in the dimer and have no direct contacts to each other.

\section{Non-crystallographic symmetry and crystal packing}

The crystal asymmetric unit contains three $\Delta^{9}$ desaturase dimers (Figure 5). In these dimers, the subunits are related by 2 -fold non-crystallographic axes which for one of the dimers is parallel to one of the crystallographic 2-fold axes, $b$. At right angles to this, parallel to $a$, there is a 3 -fold non-crystallographic screw axis relating the three 

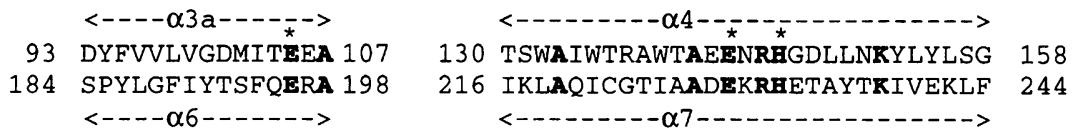

Fig. 3. Sequence alignment of the two pairs of iron binding helices based on the structural superposition. The ligands to the iron cluster are indicated by an asterisk.

Table I. Charged interactions within the subunit of $\Delta^{9}$ desaturase

\begin{tabular}{|c|c|c|c|c|}
\hline Residue & Accessible surface $\left(\AA^{2}\right)$ & Residue & Accessible surface $\left(\AA^{2}\right)$ & Comments \\
\hline E23 & 55 & $\mathrm{H} 268$ & 45 & $\mathrm{~N}$-terminal to $3_{10}$ helix after $\alpha 8$ \\
\hline $\mathrm{H} 30$ & 98 & D119 & 25 & N-terminal to loop between $\alpha 3 b$ and $\alpha 4$ \\
\hline K36 & 60 & D247 & 30 & $\alpha 1$ to loop between $\alpha 7$ and $\alpha 8$ \\
\hline E82 & 90 & $\mathrm{R} 86$ & 46 & intra $\alpha 2$ \\
\hline D92 & 31 & R159 & 38 & $\alpha 3$ a to loop between $\alpha 4$ and $\alpha 5$ \\
\hline D92 & 31 & R359 & 82 & $\alpha 3 a$ to $\beta 2$ \\
\hline D93 & 31 & R288 & 100 & $\alpha 3$ a to $\alpha 9$ \\
\hline E105 & 15 & H203 & 4 & $\alpha 3$ a to $\alpha 6$ \\
\hline D119 & 25 & R132 & 69 & loop between $\alpha 3$ and $\alpha 4$ \\
\hline D123 & 16 & R137 & 83 & loop between $\alpha 3 b$ and $\alpha 4$ to $\alpha 4$ \\
\hline E142 & 8 & R231 & 46 & $\alpha 4$ to $\alpha 7$ \\
\hline R145 & 15 & D228 & 10 & $\alpha 4$ to $\alpha 7$ \\
\hline E182 & 25 & K261 & 22 & loop between $\alpha 5$ and $\alpha 6$ to $\alpha 8$ \\
\hline R197 & 45 & E233 & 7 & $\alpha 6$ to $\alpha 7$ \\
\hline D228 & 10 & R231 & 46 & intra $\alpha 7$ \\
\hline E241 & 93 & R307 & 59 & $\alpha 7$ to $\alpha 10$ \\
\hline $\mathrm{K} 242$ & 48 & E245 & 125 & intra $\alpha 7$ \\
\hline D257 & 35 & R260 & 125 & intra $\alpha 8$ \\
\hline D257 & 35 & K261 & 22 & intra $\alpha 8$ \\
\hline $\mathrm{R} 274$ & 128 & D358 & 71 & after $\alpha 8$ to loop before $\beta 2$ \\
\hline D275 & 17 & $\mathrm{H} 281$ & 35 & after $\alpha 8$ to $\alpha 9$ \\
\hline D299 & 57 & R335 & 84 & $\alpha 10$ to $\alpha 11$ \\
\hline D325 & 72 & R329 & 167 & intra $\alpha 11$ \\
\hline R335 & 84 & E339 & 70 & intra $\alpha 11$ \\
\hline R336 & 145 & E339 & 70 & intra $\alpha 11$ \\
\hline
\end{tabular}

dimers. The translation is one third of the length of $a$, i.e. it is a local $3_{1}$ axis. The contacts between the dimers are not extensive, of the same order as other crystal contacts, and the influence of crystal contacts on the structure seems to be minor, as judged from the small deviations in noncrystallographic symmetry observed. The largest deviations are obtained for residues 336-347, where R336, E347 and/or K346 make crystal contacts, including salt bridges, in some of the subunits. The electron density in this area is weak, as mentioned above. Another area with deviations from the non-crystallographic symmetry includes residues $19-50$ which are wrapped around the subunit and also make different loose crystal contacts in the subunits. The packing of subunits, corresponding to one asymmetric unit viewed along the 3-fold and one of the 2-fold axes, is shown in Figure 5.

\section{The di-iron centre}

Previous studies have shown that $\Delta^{9}$ desaturase contains four iron atoms per dimer, and optical and Mössbauer spectroscopy indicated that these iron ions comprise a diiron-oxo cluster (Fox et al., 1993). Di-iron-oxo clusters have now been identified in a wide variety of proteins that perform both catalytic and non-catalytic functions (Nordlund and Eklund, 1995). They contain two iron atoms connected by either an oxo or hydroxo bridging ligand and have been classified based on differing primary sequence motifs providing the cluster ligands, and upon structural differences elucidated by X-ray crystallography (Fox et al., 1994; Nordlund and Eklund, 1995). Four classes have been described, one containing haemerythrin (Holmes et al., 1991) and myohaemerythrin (Sheriff et al., 1987), a second containing the R2 subunit of ribonucleotide reductase (Nordlund et al., 1990), bacterial hydrocarbon hydroxylases (Rosenzweig et al., 1993) and the $\Delta^{9}$ desaturase (Fox et al., 1994), a third containing rubrerythrin (Ravi et al., 1993; Nordlund and Eklund, 1995) and a fourth containing Fe(III)-Zn(II) purple acid phosphatase (Sträter et al., 1995) related to mammalian $\mathrm{Fe}(\mathrm{II})-\mathrm{Fe}$ (II) acid phosphatases. In addition to these soluble proteins, there is a distinct class of functionally related integral membrane proteins including fatty acid desaturases and hydrocarbon hydroxylases which contain oxygen-activated non-haem iron centres, which have yet to be structurally characterized (Shanklin et al., 1994).

The crystal structure of $\Delta^{9}$ desaturase reveals that the enzyme belongs to class II di-iron proteins and that it contains a metal cluster consistent with that proposed by Fox et al. (1994). The structure of the iron cluster is shown in Figure 6A and a view of the features surrounding the iron centre in Figure 6B. The distance between the iron ions is $4.2 \AA$ and the coordination geometry of the iron ions is a distorted octahedron where one of ligand positions is unoccupied. The structure of the cluster is highly symmetric. E143 from $\alpha 4$ and E229 from $\alpha 7$ both act as bridging ligands. E105 from $\alpha 3 \mathrm{a}$ is a bidentate ligand to one iron ion and, correspondingly, E196 from $\alpha 6$ is a bidentate ligand to the second iron ion. Each iron ion is also ligated by a nitrogen atom, $\mathrm{N} \delta 1$ in $\mathrm{H} 146$ from $\alpha 4$ and $\mathrm{H} 232$ from $\alpha 7$ respectively. The orientation of the 

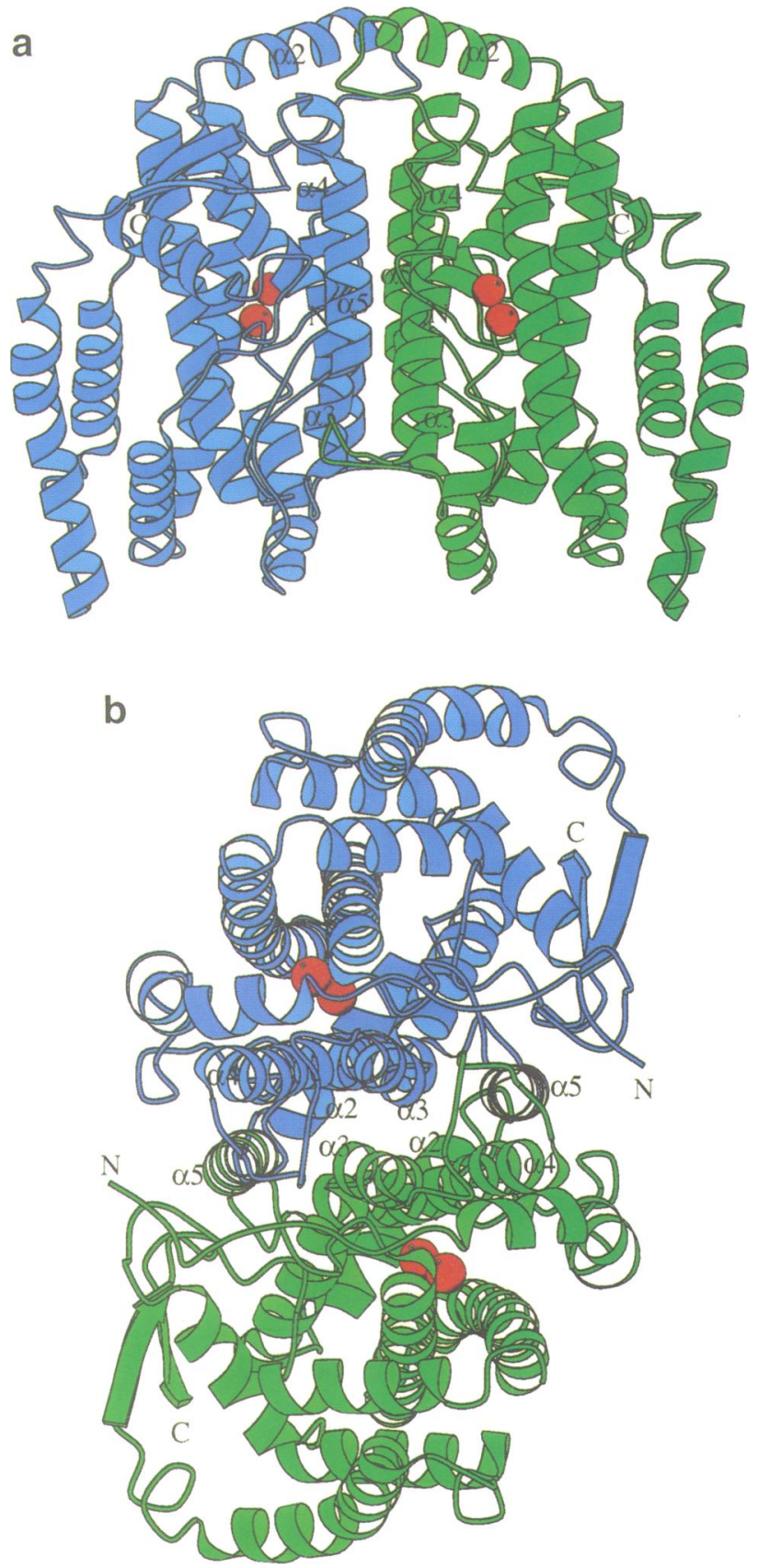

Fig. 4. View of the dimer of $\Delta^{9}$ desaturase. The two subunits are coloured green and blue, respectively and the two iron centres are indicated by red spheres. (a) Viewed along the $a$-axis and (b) $90^{\circ}$ away, along a non-crystallographic 2 -fold axis parallel to the $b$-axis.

iron ligands is, in some cases, maintained by side chain hydrogen bonds (Figure 6B); E105 interacts with H203, E143 with atom Ne1 in W139, Nع2 in H146 with the side chain of D228 which in turn interacts with the side chains of R145 and W62, NE2 in $\mathrm{H} 232$ with the side chain of E143 which in turn interacts with the side chain of R231. Further away from one of the iron ions we find atom $N \varepsilon 1$ in W139 which might be considered to be a second shell ligand. In the vicinity of the iron cluster, there is electron density corresponding to a solvent molecule. Its distances to the iron ions are 3.2 and $3.4 \AA$, respectively and it is therefore not part of the first coordination shell of the metal centre.
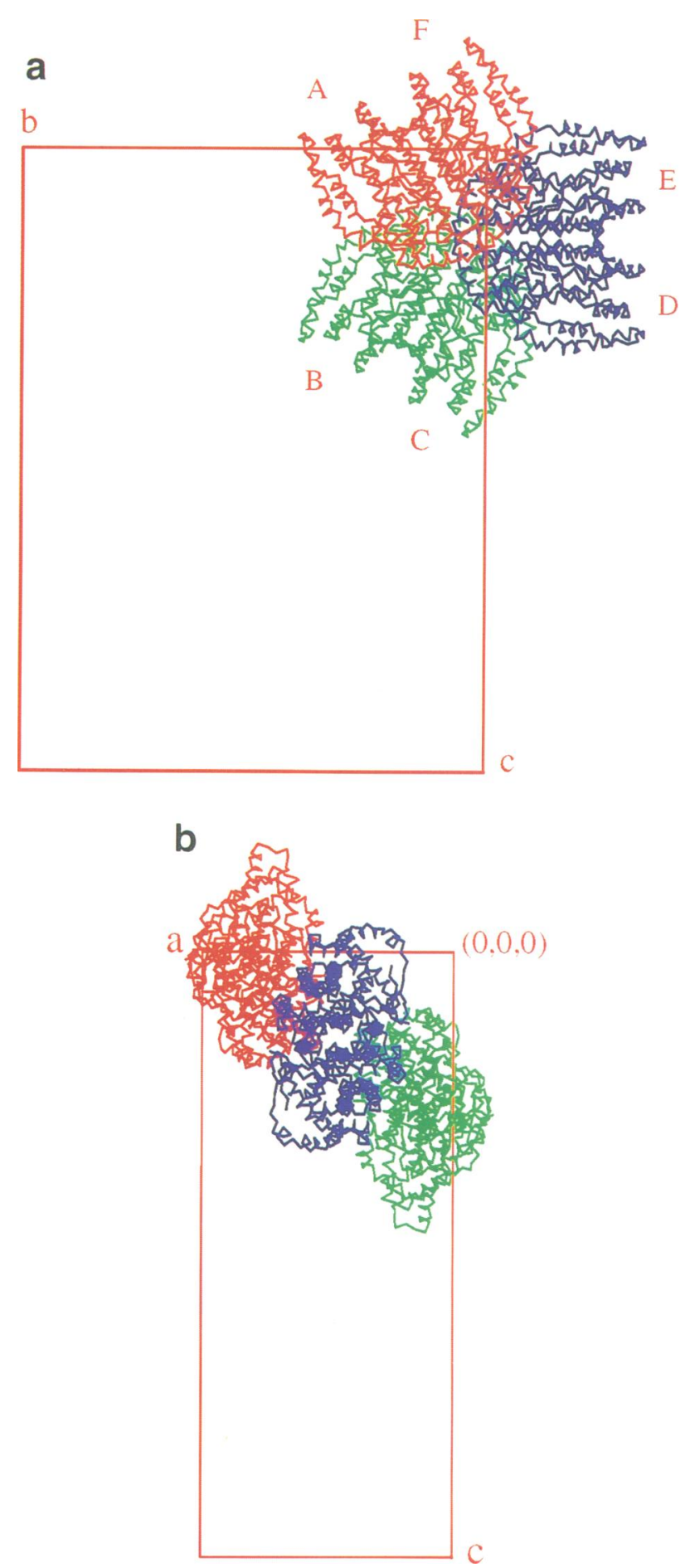

Fig. 5. Packing of dimers of $\Delta^{9}$ desaturase. The three dimers in the asymmetric unit are shown in different colours. (a) Viewed along the non-crystallographic $3_{1}$ axis (and the $a$-axis). The subunits are related by a local 2 -fold symmetry axis, located perpendicular to the noncrystallographic 3 -fold screw axis. (b) Viewed $90^{\circ}$ away, along the local 2 -fold and $b$-axis.

\section{Form of the desaturase in the crystal structure}

The presence of a $\mu$-oxo bridged di-iron cluster in the diferric state of $\Delta^{9}$ desaturase has been demonstrated unambiguously using Mössbauer and resonance Raman spectroscopy (Fox et al., 1993, 1994). It is surprising, therefore, that we do not observe a $\mu$-oxo bridge in the 
a
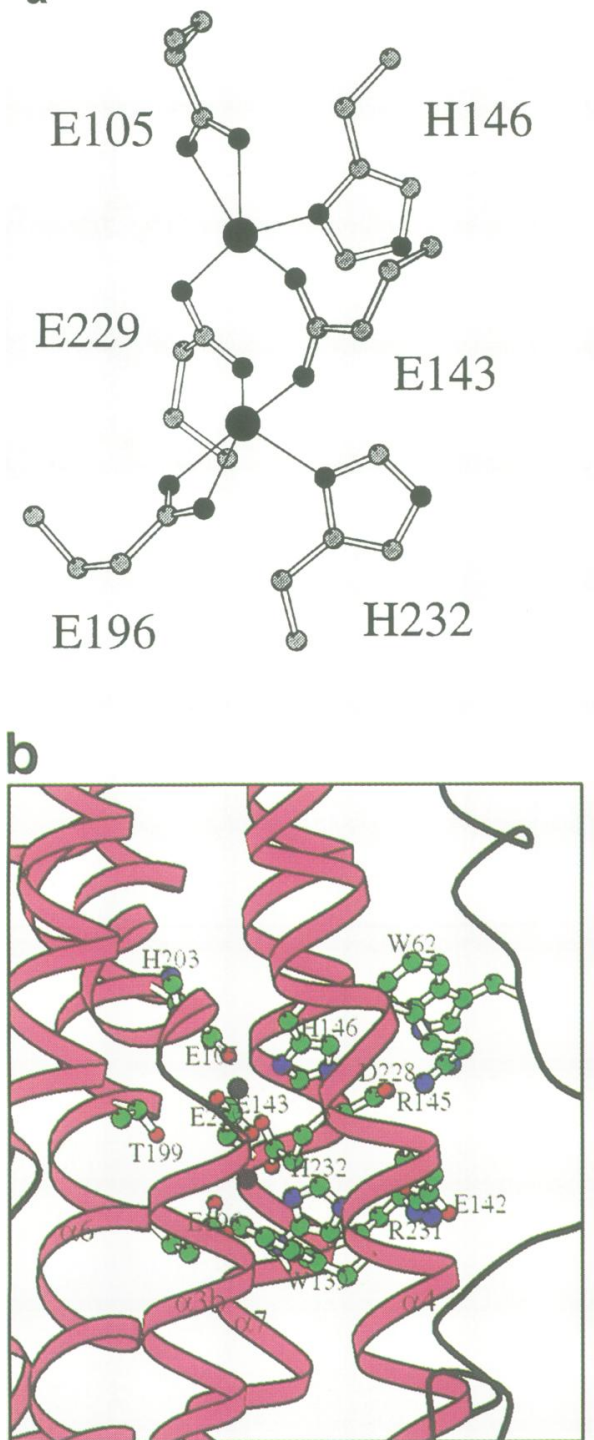
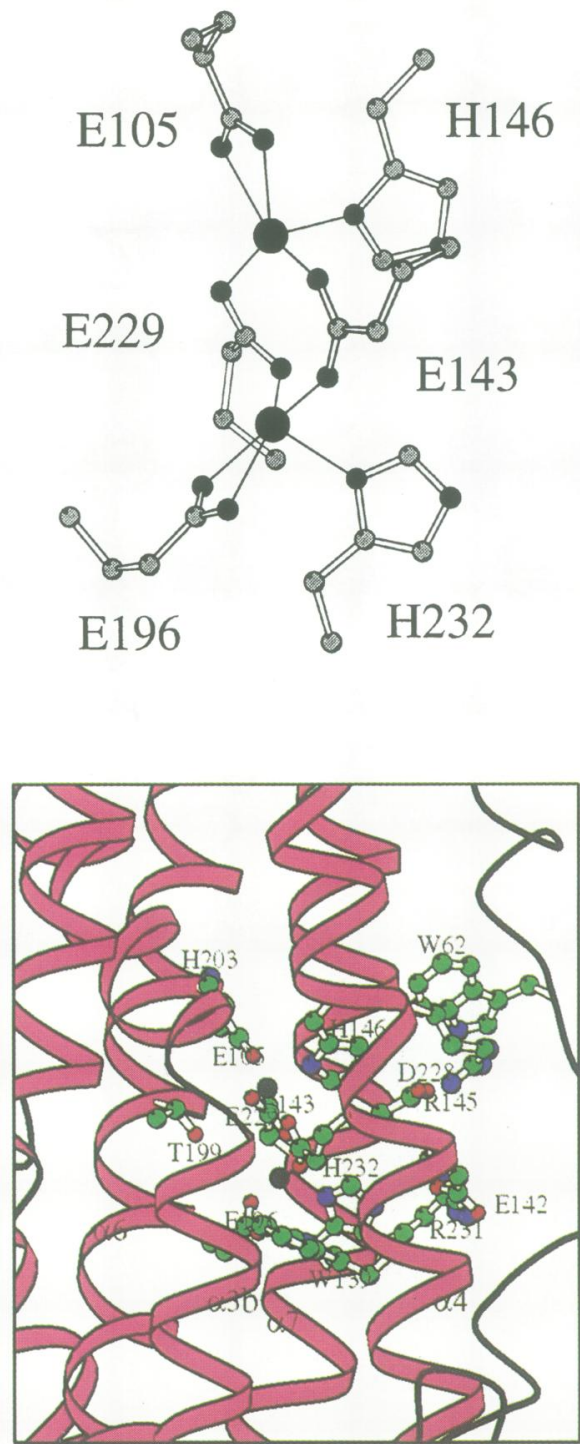

Fig. 6. (a) Stereo view of the di-iron centre with ligands. (b) Stereo view of the di-iron centre and its local environment.

electron density map of $\Delta^{9}$ desaturase because the enzyme used for the experiments was in the oxidized state and no reducing agents were added to the mother liquor. In addition, the distance between the iron ions $(4.2 \AA)$ is longer than expected for a di-iron cluster with an intact $\mu$-oxo bridge. In the oxidized form of ribonucleotide reductase with the $\mu$-oxo bridge present, the iron-iron distance is $3.3 \AA$ (Nordlund and Eklund, 1993). The geometry observed in $\Delta^{9}$ desaturase is strikingly similar to that seen in the reduced form of ribonucleotide reductase, where, upon chemical reduction of $\mathrm{R} 2$, the distance between the iron ions is increased to $3.8 \AA$, the $\mu$-oxo bridge is lost and the ligand arrangement becomes very symmetric as shown by protein crystallography (Nordlund and Eklund, 1995) and Mössbauer spectroscopy (Lynch et al., 1989). We suggest that exposure of the desaturase crystals to X-ray radiation results in photochemical reduction of the metal centre which is accompanied by loss of the $\mu$-oxo bridge and ligand rearrangement. Thus, the structure of the $\Delta^{9}$ desaturase presented here most likely represents the reduced form of the enzyme. The crystal structure of the $\Delta^{9}$ desaturase reveals a highly symmetric ligand arrangement of the iron cluster in the diferrous form of the enzyme, in agreement with resonance Raman studies (Fox et al., 1994). Deviations in the symmetric ligand arrangement in the reduced state of the enzyme, as suggested from previous temperature-dependent Mössbauer data (Fox et al., 1994), might be due to variations in bond lengths and bond angles in the two metal sites, too small to be observable in the electron density maps at the current resolution.

\section{Active site and interactions with other proteins}

The structure of $\Delta^{9}$ desaturase described here is very likely that of the diferrous form of the enzyme that results from interaction of $\Delta^{9}$ desaturase and ferredoxin in vivo. From the three-dimensional structure, two possible routes for electron transfer from the surface to the iron centre can be postulated. One of these extends along the axis of the helix bundle and involves the structurally consecutive cluster of aromatic side chains of W139, W135, Y236, F189 and W132. The NE1 atom of W139 is rather close 




Fig. 7. View of the postulated substrate channel in $\Delta^{9}$ desaturase. The position of the di-iron cluster is illustrated by the two pink spheres. Side chains coordinating to the di-iron cluster and forming the predominantly hydrophobic substrate binding pocket are shown. A model of the substrate, stearic acid, is fitted into this pocket. The picture was generated using VOIDOO (Kleywegt and Jones, 1994) and O (Jones et al., 1991).

to one of the irons, and the Nel atom of W132 points towards the surface of the subunit close to the protruding loop between $\alpha 3 \mathrm{~b}$ and $\alpha 4$. This loop and the cap part of $\alpha 1$ could then form a possible interaction surface for the ferredoxin molecule. Another possible route for electron transfer from the surface to the iron centre involves residues W62, D228 and H146, analogous to what has been suggested for R2 (Nordlund and Eklund, 1993). This pathway leads to the flat surface formed by helices $\alpha 1$, $\alpha 6, \alpha 7, \alpha 10$ and $\alpha 11$.

The solvent molecule bound in the vicinity of the iron centre is located in a small, hydrophobic pocket, and the closest amino acid side chains to this solvent molecule are T199 and W139. A similar cavity, with a Thr side chain at the equivalent position, has been found in MMO, and it has been suggested that this cavity could provide a suitable binding site for the oxygen molecule (Rosenzweig et al., 1993). The side chain of T213 in MMO has been implicated to be involved in oxygen activation (Rosenzweig et al., 1993) in a manner similar to residue T252 in cytochrome P450 (Martinis et al., 1989).

Because the iron centre is buried in the interior of the $\Delta^{9}$ desaturase, we would expect to find a substrate cleft lined with hydrophobic residues connecting the surface of 
the enzyme to the active site. Indeed, a narrow, very deep channel can be found extending from the surface far into the protein (Figure 7). The channel passes the di-iron centre on the same side as the proposed oxygen binding site. At the bottom of this channel we find the side chain of L115, and the walls consist of residues W139, T192, Y111, M114, Y191, Q195, P266, T199 and T104. The channel then passes the iron cluster and continues towards the surface with residues Y292, M265, F279 and S283 at the narrow entrance of this cleft. The overall shape of the substrate channel which is bent at the position of the iron cluster facilitates binding of the product, oleoyl-ACP, with cis configuration at the double bond.

After refinement, we found strong, elongated electron density in the averaged $2 F_{0}-F_{\mathrm{c}}$ electron density maps in this channel which had not been assigned to solvent or protein atoms. Based on the shape of this density and the hydrophobic character of the pocket, we suggest that this electron density may represent the hydrophobic acyl tail of a $\beta$-octylglucoside molecule. The hydrocarbon tail of the octylglucoside would fit well in this density, but the density corresponding to the sugar moiety is poorly defined. This putative octylglucoside molecule is oriented with its tail deep down in the hydrophobic pocket close to the di-iron cluster and the carbohydrate moiety extending towards the surface. The weak electron density for this part of the molecule might indicate local disorder resulting from less specific interactions with the enzyme.

Modelling of a stearic acid in the presumed substrate binding pocket renders the $\mathrm{C} 9$ carbon atom at about $5.5 \AA$ from one of the iron ions (Figure 7). This carbon atom, where the double bond will be formed, is also close to the small pocket with the bound solvent molecule, in fact the water molecule is bridging the distance between the C9 carbon of the substrate and the closest iron ion. We suggest that, in the active enzyme, this pocket will be occupied by an oxygen molecule bound to one or both of the iron atoms. During catalysis, a peroxide radical could be generated capable of abstracting one of the hydrogen atoms at the $\mathrm{C} 9$ position of the fatty acid.

\section{Comparison with other di-iron proteins}

A superposition of the structure of $\Delta^{9}$ desaturase on the three-dimensional structures of two other di-iron proteins, the R2 subunit of ribonucleotide reductase from Escherichia coli (Nordlund et al., 1990) and the $\alpha$ subunit of MMO from Methylococcus capsulatus (Rosenzweig et al., 1993) shows that the overall structures are rather similar, with an r.m.s. fit of $1.90 \AA$ for $144 \mathrm{C} \alpha$ atoms $\left(\Delta^{9}\right.$ desaturase versus R2) and an r.m.s. fit of $1.98 \AA$ for 117 equivalent $\mathrm{C} \alpha$ atoms $\left(\Delta^{9}\right.$ desaturase versus MMO). The folds are very similar, most of the $\alpha$-helices, $\alpha 1-\alpha 8$ and $\alpha 10$ have their counterpart in R2 and MMO. Amino acids which are structurally equivalent are aligned in Figure 8. There are few conserved amino acids besides the iron ligands but there can be little doubt that these enzymes are evolutionary related.

There are significant differences in the structure of the iron centres in the three proteins. In general, the metal centre in $\Delta^{9}$ desaturase is considerably more symmetrical than in the two other proteins. However, when compared with the structure of the reduced form of R2 (Nordlund and Eklund, 1995), the coordination geometries of the dinuclear iron centre in $\Delta^{9}$ desaturase and R2 are more similar. The most significant difference is that, in $\Delta^{9}$ desaturase, the terminal carboxylates E105 and E196, respectively, act as bidentate ligands, whereas in $\mathrm{R} 2$, the equivalent side chains are monodentate ligands to the iron ions.

$\mathrm{R} 2$ is unique among these enzymes in that it forms a stable radical at position Y122. The corresponding residue in $\Delta^{9}$ desaturase is $\mathrm{L} 150$, located in the hydrophobic cluster making packing interactions in the four helix bundle binding the iron cluster, and no evidence is available which might indicate that this residue is required for catalytic activity.

There are only very few amino acid residues which are conserved in all three enzymes (Figure 8). Amongst those are the ligands to the metal ions, with the exception of E105 which is replaced by an aspartic acid in R2. The only other invariant residues are I225 and D228. Residue I225 is in the vicinity of the di-iron cluster (closest distance $4.6 \AA$ ) on the opposite side of the substrate channel. The side chain is packed between H203, H146 and W62 in the three-dimensional structure, and a more detailed examination of its function has to await the results from site-directed mutagenesis studies. The other invariant protein residue in the three enzymes, D228, is part of an electron transfer pathway from the dinuclear iron centre to the surface of the protein which has been suggested for R2 (Nordlund and Eklund, 1993). In R2, this pathway runs from one of the iron ions via the side chain of $\mathrm{H} 118$, D237 to W48, which is located at the surface of the protein. These residues are conserved in $\Delta^{9}$ desaturase, and a similar pathway for electron transfer can be postulated including the structurally equivalent residues H146, D228 and W62, as mentioned before. Furthermore, a slightly modified pathway for electron transfer could also be suggested for MMO. In this case, the iron ligand (H147) and the aspartic acid residues (D242) are conserved; however, the structure at the surface is different. Nevertheless, an aromatic side chain (Y67) at the surface is in the vicinity of the side chain of D242.

Most of the other residues conserved between $\Delta^{9}$ desaturase and R2 on one hand and $\Delta^{9}$ desaturase and MMO on the other hand are located at the surface of the protein, or involved in packing interactions. Conserved residues common to both $\mathrm{R} 2$ and $\Delta^{9}$ desaturase in the proximity of the di-iron site are residues W135 and W139. While W135 and W139 are strictly conserved in the desaturases, the corresponding residues W107 and W111 in R2 are not strictly conserved. Except for the T4 and E.coli protein, W135 is replaced by a phenylalanine or a tyrosine side chain. Similarly, W139 is replaced by a glutamine residue.

\section{Conclusions}

The castor $\Delta^{9}$ desaturase is the first fatty acid desaturase enzyme for which an X-ray crystallographic structure has been determined. The castor $\Delta^{9}$ desaturase is an appealing model enzyme for identifying residues required for catalysis and electron transport for this class of di-iron proteins because, unlike R2 and MMO, it is active as a homodimer and, therefore, does not require additional protein components. In this regard, we have devised a bacterial system that can be used for rapid screening of desaturase mutants 
$\alpha 1$

19 MPPREVHVQVTH्HSMPPQKIEIF́KSLDNWAEENंILVHLKPVEK 60

DIFEKLI

$\stackrel{\text { ANET }}{\mathrm{53} \longrightarrow \mathrm{YARM}}$

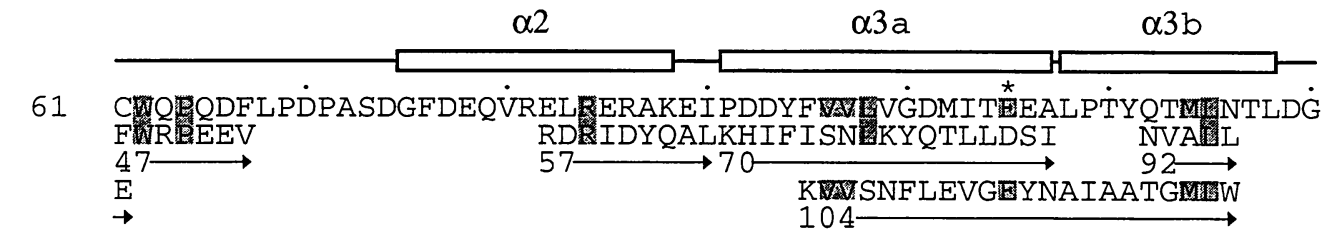

$\alpha 4$

$\alpha 5$

121 VRDETGASPTSWAIRTR PELETUVETAFSEITI SRSYTHIIRNIV

102

AEQKNGYLQVLDEIRTHQCAYVNYYFA

131

$\alpha 6$

$\alpha 7$

181 TENSP

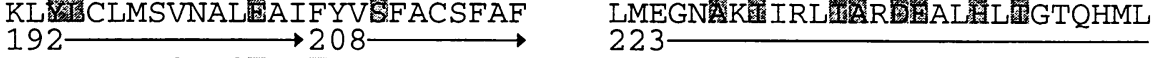

QLVGEAFWNPLIVAVTEWAAANATEITPTVFLSWETEELRHM

$205 \longrightarrow 212 \longrightarrow$
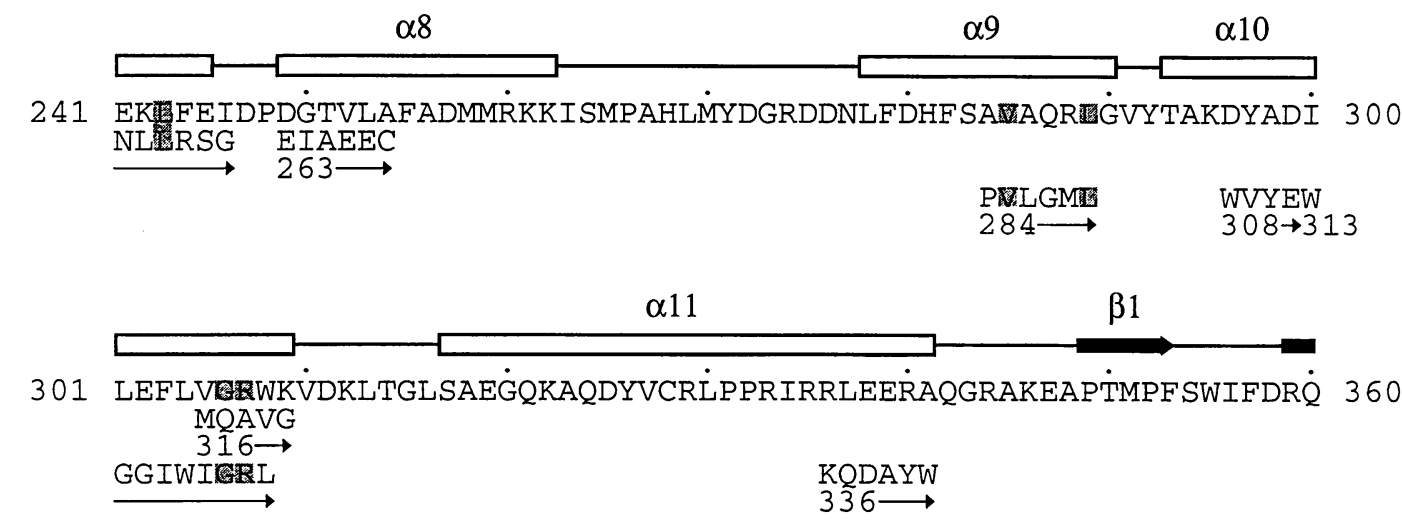

$\beta 2$

361 VKL 363

Fig. 8. Amino acid sequence alignment of structurally equivalent residues in $\Delta^{9}$ desaturase, the R2 protein of ribonucleotide reductase and MMO Pairwise conserved amino acid residues are shaded. The ligands to the di-iron clusters are indicated by an asterisk. First line, secondary structure elements in $\Delta^{9}$ desaturase: second line, amino acid sequence of $\Delta^{9}$ desaturase: third line. amino acid sequence of R2: and fourth line. amino acid sequence of MMO.

for functionality (Cahoon et al., 1996; E.B.Cahoon and J.Shanklin, unpublished results). In addition, the castor $\Delta^{9}$ desaturase is a member of a family of highly homologous enzymes that differ with respect to chain length specificity and regiospecificity. Understanding the architecture of the substrate channel with respect to the iron centre will be a critical step towards our goal of determining the molecular factors that govern such differences. This information will be used for the rational re-design of desaturase enzymes with desired chain length and regiospecificities. Resulting enzymes may be introduced into crop plants to alter their storage lipid composition in desired ways.

\section{Materials and methods}

\section{Enzyme purification and crystallization}

Recombinant $\Delta^{9}$ desaturase was expressed in E.coli cells and purified as described previously (Fox et al., 1993). Crystallization of the enzyme was achieved according to Schneider et al. (1992) with slight modifications. Enzyme samples were concentrated to $12-18 \mathrm{mg} / \mathrm{ml}$. A $7.5 \mu \mathrm{l}$ aliquot of protein solution was mixed with the same amount of 
Table II. Data collection and phasing statistics

\begin{tabular}{|c|c|c|c|c|c|}
\hline \multirow[t]{2}{*}{ Data set } & \multirow[t]{2}{*}{ No. of crystals } & \multirow[t]{2}{*}{ Resolution ( $\AA$ ) } & \multicolumn{2}{|c|}{ Data collection ( $\%$ of total) } & \multirow[t]{2}{*}{$R_{\text {merge }}{ }^{\mathrm{a}}$} \\
\hline & & & Measured reflections & Unique reflections & \\
\hline NAT1 & 1 & 3.1 & 101163 & $38774(88 \%)$ & 0.066 \\
\hline NAT $2^{b}$ & 2 & 2.4 & 403539 & $79674(87 \%)$ & 0.075 \\
\hline $\mathrm{KAu}(\mathrm{CN})_{2}$ & 1 & 3.2 & 101390 & $35290(87 \%)$ & 0.081 \\
\hline \multirow[t]{2}{*}{ Derivative } & \multirow[t]{2}{*}{ No. of sites } & \multicolumn{3}{|l|}{ Phasing statistics } & \\
\hline & & $\overline{R_{\text {deriv }}{ }^{c}}$ & $R_{\text {cullis }}{ }^{\mathrm{d}}$ & Phasing power ${ }^{e}$ & \\
\hline $\mathrm{KAu}(\mathrm{CN})_{2}$ & 18 & 0.26 & 0.88 & 1.02 & \\
\hline
\end{tabular}

${ }^{\mathrm{a}} R_{\text {merge }}=\Sigma_{\mathrm{hkl}} \Sigma_{\mathrm{i}} \mid I_{\mathrm{i}}-\langle I>| / \Sigma_{\mathrm{hkl}} \Sigma_{\mathrm{i}}<I>$ where $I_{\mathrm{i}}$ are the intensity measurements for a reflection and $\langle I\rangle$ is the mean value for this reflection. ${ }^{b}$ Data set NAT2 was collected at beamline X12-C at NSLS, Brookhaven.

${ }^{c} R_{\text {deriv }}=\Sigma\left|F_{\mathrm{PH}}-F_{\mathrm{P}}\right| / \Sigma|F \mathrm{P}|$ where $F_{\mathrm{PH}}$ is the structure factor amplitude of the derivative crystal and $F_{\mathrm{P}}$ is that of the native.

${ }^{\mathrm{d}} R_{\text {Cullis }}=\Sigma\left\|F_{\mathrm{PH}} \pm F_{\mathrm{P}}|-| F_{\mathrm{H}(\mathrm{calc})}\right\| / \Sigma\left|F_{\mathrm{PH}}-F_{\mathrm{P}}\right|$, where $F_{\mathrm{PH}}$ and $F_{\mathrm{P}}$ are defined as above and $F_{\mathrm{H}(\mathrm{calc})}$ is the calculated heavy atom structure factor amplitude summed over centric reflections only.

${ }^{\text {e}}$ Phasing power $=F(\mathrm{H}) / \mathrm{E}$, the r.m.s. heavy atom structure factor amplitudes divided by the lack of closure error.

the reservoir solution, placed on coverslips and allowed to equilibrate over $1 \mathrm{ml}$ of the well solution at $20^{\circ} \mathrm{C}$. The reservoir solution contained $0.08 \mathrm{M}$ cacodylate buffer $\mathrm{pH} 5.4,200 \mathrm{mM} \mathrm{Mg}$ acetate, $75 \mathrm{mM}$ ammonium sulfate, $2 \mathrm{mM} \mathrm{LiCl}, 1 \mathrm{mM} \mathrm{KCl}, 0.2 \% \beta$-octyl glucoside and $12-15 \%$ PEG 4000 as precipitant. The crystals were orthorhombic, space group $\mathrm{P} 2{ }_{1} 2{ }_{1} 2$, with cell dimensions $a=82.2, b=147.8$ and $c=198.2 \AA$.

\section{Data collection and preparation of heavy metal derivatives}

Attempts to prepare heavy metal derivatives by soaking native crystals of the enzyme with solutions of various heavy metal salts in mother liquor were not very successful. Most soaking experiments resulted in crystal cracking or non-isomorphous crystals, and only one useful heavy metal derivative could be prepared by soaking desaturase crystals in mother liquor containing $5 \mathrm{mM} \mathrm{KAu}(\mathrm{CN})_{2}$ for 1 week. X-ray data from native and derivative crystals were collected on a UCSD multi-wire area detector system (Hamlin, 1985) at the Department of Molecular Biology, Uppsala. Measured frames were processed with MADNES (Pflugrath, 1987). A second native data set was collected at beamline X12-C at NSLS, Department of Biology, Brookhaven National Laboratory. Data frames were collected as $1^{\circ}$ oscillations using a MAR Research image plate. The data frames were processed with DENZO and scaled with SCALEPACK (Otwinowski, 1993). Details of the data collection statistics are given in Table II.

\section{Phase determination, model building and crystallographic refinement}

Most crystallographic calculations were done using the CCP4 program suite (CCP4, 1994). The initial crystallographic analysis was carried out with the data sets collected on the multi-wire detector to $3.1 \AA$ resolution. The difference Patterson map for the gold derivative was analysed using RSPS (Knight, 1989). Two sites, related by a strong cross-peak in the difference Patterson map, were used for calculation of difference Fourier maps and new sites were identified. Finally, six main sites and 12 minor sites were found and the heavy metal parameters were refined using MLPHARE (see Table II). From results of the rotation function calculations and the positions of the metal ions, the direction and position of the local symmetry operators, relating the six subunits of $\Delta^{9}$ desaturase in the asymmetric unit, could be determined. Six-fold non-crystallographic symmetry averaging using the RAVE program (Jones, 1992) and a spherical envelope, centred at the presumed position of one $\Delta^{9}$ desaturase subunit, was then used to refine the initial SIR phases. From an electron density map at low resolution, based on these phases, part of the central four helix bundle, coordinating the di-iron centre and the iron atoms could be identified. The coordinates of the iron atoms were refined from the anomalous native data, and new phases were calculated based on the Au derivative and the anomalous contribution from the iron atoms. A new envelope for the subunit was made using MAMA (Kleywegt and Jones, 1994) by approximately orienting a subunit of R2 at the correct position for the helix bundle.

After non-crystallographic averaging, it was possible to build a starting model of the desaturase from the electron density map. Cycles of model building, refinement in XPLOR (Brünger, 1989, 1990), redefinition of the envelope, refinement of the symmetry operators using IMP (Kleywegt and Jones, 1994) and averaging were performed until no new electron
Table III. Statistics for the final model of $\Delta^{9}$ desaturase after crystallographic refinement

\begin{tabular}{ll}
\hline $\begin{array}{l}\text { Resolution range } \\
\text { No. of reflections }\end{array}$ & $7.0-2.4 \AA$ \\
$\quad$ in working set & 77044 \\
in test set & 1928 \\
$R_{\text {cryst }}$ & $22.0 \%$ \\
$R_{\text {free }}$ & $28.5 \%$ \\
No. of amino acid residues in the asymmetric unit & 2070 \\
Non-hydrogen protein atoms & 16776 \\
Iron atoms & 12 \\
Solvent atoms & 538 \\
Mean $B\left(\AA^{2}\right)$ & $60.0 \AA^{2}$ \\
R.m.s. $B\left(\AA^{2}\right)$ & $2.3 \AA^{2}$ \\
R.m.s. bond length $(\AA)$ & 0.008 \\
R.m.s. bond angles $\left({ }^{\circ}\right)$ & 1.34 \\
Average real-space-fit value & 0.76 \\
Ramachandran plot: percentage of non-glycine & $91.3(0.3)$ \\
residues in favourable (disallowed) regions &
\end{tabular}

density appeared in the averaged maps. At this stage, one more loop, which seemed to have a different structure in the subunits, was built from the $2 F_{0}-F_{\mathrm{c}}$-maps.

Crystallographic refinement was carried out with XPLOR, using the Engh and Huber force field (Engh and Huber, 1991) and noncrystallographic symmetry restraints. Due to the low resolution (3.1 $)$ of the data set, an overall $B$-value was used. The model at this stage had a crystallographic $R$-factor of $26.7 \%$ with 6 -fold non-crystallographic symmetry restraints imposed in the refinement. At this stage of the refinement, a new native data set to $2.4 \AA$ resolution collected at NSLS became available and refinement continued with this data set. The process of refinement was monitored by $2.5 \%$ of the reflections which were not included in the refinement but were used to calculate $R_{\text {free }}$ (Brünger, 1992).

Even at the resolution of $2.4 \AA$, the observation to parameter ratio is just about one and the refinement problem is ill determined. Therefore, during the whole refinement process, non-crystallographic symmetry restraints were employed in order to avoid over-fitting of the diffraction data. Only those parts of the structure which were not restrained were, from the averaged electron density maps, those judged not to obey the non-crystallographic symmetry. This includes residues 19-50, 121-122, $127-129,208-212,241-253,259-260,308-319,336-348$ and some side chains. The electron density for some residues in the region 336347 is so weak that their positions must be considered arbitrary and the occupancies for these atoms were therefore set to zero. Overall anisotropic refinement lowered the free $R$-factor by $\sim 2 \%$. At this stage, water molecules were added to the model. Individual $B$-factors were also refined but restrained by the non-crystallographic symmetry. The final model has a crystallographic $R$-factor of $22.0 \%\left(R_{\text {free }} 28.5 \%\right)$. The r.m.s. deviations for the restrained $C \alpha$ positions (263 atoms) of the subunit $A$ to the corresponding parts of the other subunits are $0.06 \AA$ and for all 
$\mathrm{C} \alpha$ atoms (345 atoms) $0.26,0.23,0.24,0.32,0.25 \AA$. respectively. Statistics for the final model are given in Table III.

The protein model was analysed using the PEPFLIP and RSFIT options in O (Jones et al.. 1991) and with the program PROCHECK (Laskowski et al.. 1993). The atomic coordinates will be deposited with the Brookhaven Protein Data Bank.

\section{Structural comparisons}

All structural superpositions were performed by least-squares methods using O (Jones et al.. 1991) and were done pairwise. Superposition was done by selecting an initial set of equivalent $\mathrm{C} \alpha$ atoms consisting of four stretches of the polypeptide chain ( $\sim 10$ residues each) from the four helices containing the ligands to the di-iron centre. This initial alignment was maximized subsequently by including all $C \alpha$ atoms from the atomic models. Residues were considered structurally equivalent if they were within $3.8 \AA$ from each other and within a consecutive stretch of more than three equivalent residues.

\section{Acknowledgements}

We thank the staff, in particular R.M.Sweet, at the Department of Biology. Brookhaven National Laboratory, for access to beamline X12-C at NSLS. We thank Hans Eklund, Uppsala for the atomic coordinates of the R2 subunit of ribonucleotide reductase. and Pär Nordlund. Stockholm for the atomic coordinates for methane monooxygenase. This work was supported by grants from the Swedish Natural Science Research Council and the Hagberg Foundation (to Y.L.) and the Office of Basic Energy Research of the U.S. Department of Energy (to J. S.). G.S. acknowledges receipt of a major equipment grant from the Knut and Alice Wallenberg Foundation.

\section{References}

Barlow.D.J. and Thornton.J.M. (1983) Ion pairs in proteins. J. Mol. Biol.. 168, 857-885.

Brändén.C.-I. and Jones.T.A. (1990) Between objectivity and subjectivity. Nature. 343, 687-690.

Brünger.A. (1989) Crystallographic refinement by simulated annealing: application to crambin. Acta Crystallogr. A45, 50-61.

Brünger.A. (1990) The X-PLOR Manual. Yale University. New Haven. CT.

Brünger.A. (1992) Free R value: a novel statistical quantity for assessing the accuracy of crystal structure. Nature, 355, 472-475.

Cahoon.E.B.. Shanklin.J. and Ohlrogge.J.B. (1992) Expression of a coriander desaturase results in petroselinic acid production in transgenic tobacco. Proc. Natl Acad. Sci. USA. 89. 11184-11188.

Cahoon.E.B.. Becker.C.K., Shanklin.J. and Ohlrogge.J.B. (1994a) cDNAs for isoforms of the delta 9-stearoyl-acyl carrier protein desaturase from Thunbergia alata endosperm. Plant Physiol.. 106. 807-808.

Cahoon.E.B.. Cranmer.A.M.. Shanklin.J. and Ohlrogge.J.B. (1994b) Delta 6 hexadecenoic acid is synthesized by the activity of a soluble delta 6 palmitoyl-acyl carrier protein desaturase in Thunbergia alata. J. Biol. Chem.. 269. 27519-27526.

Cahoon.E.B.. Mills.L.A. and Shanklin.J. (1996) Modification of the fatty acid composition of Escherichia coli by coexpression of plant acylcarrier protein desaturase and ferredoxin. J. Bacteriol.. 178, 936-939.

Collaborative Computational Project. Number 4 (1994) The CCP4 suite: programs for protein crystallography. Acta Crystallogr. D50. 760-763.

Engh.R.A. and Huber.R. (1991) Accurate bond and angle parameters for X-ray protein structure refinement. Acta Crystallogr. A47. 392-400.

Fox.B.G.. Shanklin.J.. Somerville.C. and Münck.E. (1993) Stearoyl-acyl carrier protein $\Delta 9$ desaturase from Ricinus communis is a diiron-oxo protein. Proc. Natl Acad. Sci. USA. 90, 2486-2490.

Fox.B.G.. Shanklin.J.. Ai.J.. Loehr,T.M. and Sanders-Loehr.J. (1994) Resonance Raman evidence for an Fe-O-Fe center in stearoyl-ACP desaturase. Primary sequence identity with other diiron-oxo proteins Biochemistry. 33. 12776-12786.

Gibson.K.J. (1993) Palmitoleate formation by soybean stearoyl-acyl carrier protein desaturase. Biochim. Biophys. Acta. 1169. 231-235.

Hamlin.R. (1985) Multiwire area X-ray diffractometers. Methods En-imol. 114. 416-452.

Harwood.J.L. (1988) Fatty acid metabolism. Annu. Rev: Plant Physiol. Plant Mol. Biol.. 39. 101-138.
Holmes.M.A.. Le Trong.I.. Turley.S.. Sieker.L.C. and Stenkamp.R.E. (1991) Structures of deoxy and oxy hemerythrin at $2.0 \AA$ A resolution. J. Mol. Biol.. 218. 583-593.

Jones.T.A. (1992) a, yaap. asap. @\#*? A set of averaging programs. In Dodson.E.J.. Gover.S. and Wolf.W. (eds), CCP4 Study Weekend 1992: Molecular Replacement. Daresbury Laboratory. Daresbury. UK. pp. 91-105.

Jones.T.A., Zou.J.-Y.. Coman.S. and Kjeldgaard.M. (1991) Improved methods for building protein models into electron density maps and the location of errors in these models. Acta Crystallogr.. A47. 100-119.

Kleywegt.G. and Jones,T.A. (1994) Detection, delineation. measurement and display of cavities in macromolecular structures. Acta Crystallogr. D50. 178-185.

Knight.S. (1989) Ribulose-1.5-bisphosphate carboxylase/oxygenase: a structural study. PhD Thesis. Swedish University of Agricultural Sciences. Uppsala.

Kraulis.P. (1991) Molscript: a program to produce both detailed and schematic plots of protein structures. J. Appl. Cristallogr.. 24. 946950 .

Laskowski.R.A.. McArthur.M.W.. Moss.D.S. and Thornton.J.M. (1993) PROCHECK: a program to check the quality of protein structures. J. Appl. Crystallogr. 26. 282-291.

Lynch.J.B.. Juarez.G.C. Münck.E. and Que.L..Jr (1989) Mössbauer and EPR studies of the binuclear iron centre in ribonucleotide reductase from Escherichia coli. J. Biol. Chem.. 264. 8091-8096.

Martinis.S.A.. Atkins.W.A.. Stayton.P.S. and Sligar.S.G. (1989) A conserved residue of cytochrome P-450 is involved in heme-oxygen stability and activation. J. Am. Chem. Soc., 111, 9252-9253.

McKeon.T.A. and Stumpf.P.K. (1982) Purification and characterization of the stearoyl-acyl carrier protein. J. Biol. Chem.. 257. 121+1-12147.

Nagai.J. and Bloch.K. (1966) Enzymatic desaturation of stearoyl acyl carrier protein. J. Biol. Chem.. 241. 1925-1927.

Nagai.J. and Bloch.K. (1968) Enzymatic desaturation of stearoyl acyl carrier protein. J. Biol. Chem.. 243. 4626-4633.

Nordlund.P. and Eklund.H. (1993) Structure and function of the Escherichia coli ribonucleotide reductase protein R2. J. Mol. Biol.. 232. 123-164.

Nordlund.P. and Eklund.H. (1995) Di-iron-carboxylate proteins. Curr: Opin. Struct. Biol.. 5, 758-766.

Nordlund.P.. Sjöberg.B.-M. and Eklund.H. (1990) Three-dimensional structure of the free radical protein of ribonucleotide reductase. Nature, 345. 592-598.

Nordlund.P.. Dalton.H. and Eklund.H. (1992) The active site structure of methane monooxygenase is closely related to the binuclear iron centre of ribonucleotide reductase. FEBS Lett.. 307. 257-262.

Ohlrogge.J.B. (1994) Design of new plant products: engineering of fatty acid metabolism. Plant Phisiol.. 104. 821-826.

Ohlrogge.J.B. and Browse.J.A. (1995) Lipid biosynthesis. Plant Cell. 7. 957-970.

Otwinowski.Z. (1993) Proceedings of the CCP4 Study. Weekend. Daresbury Laboratory. Warrington. UK. pp. 56-62.

Pflugrath.J. (1987) MADNES: Munich area detector NE system. Users Guide. Cold Spring Harbor Laboratory Press. Cold Spring Harbor. NY.

Ravi.N.. Prichrel.B.C., Kurtz,D.M. and Huynh.B.H. (1993) Spectroscopic characterization of Fe-reconstituted rubrerythrin, a non-heme iron protein with structural analogies to ribonucleotide reductase. Biochemistry: 32. 8487-8491

Rosenzweig.A.C.. Frederick.C.A.. Lippard.S.J. and Norlund.P. (1993) Crystal structure of a bacterial non-haem iron hydroxylase that catalyses the biological oxidation of methane. Nature, 366. 537-540.

Schneider.G.. Lindqvist.Y.. Shanklin.J. and Somerville.C. (1992) Preliminary crystallographic data for stearoyl-acyl carrier protein desaturase from castor seed. J. Mol. Biol. 225. 561-564.

Shanklin.J. and Somerville.C. (1991) Stearoyl-acyl-carrier-protein desaturase from higher plants is structurally unrelated to the animal and fungi homologs. Proc. Natl Acad. Sci. USA. 88. 2510-2514.

Shanklin.J.. Whittle.E. and Fox.B.G. (1994) Eight histidine residues are catalytically essential in a membrane-associated iron enzyme. stearoyl$\mathrm{COA}$ desaturase, and are conserved in alkane hydroxylase and xylene monooxygenase. Biochemistry, 33, 12787-12794.

Sheriff,S.. Hendrickson.W.A. and Smith.J.L (1987) Structure of myohemerythrin in the azidomet state at $1.7 / 1.3 \AA$ A resolution. J. Mol. Biol. 197. 273-296.

Somerville.C. and Browse.J. (1991) Plant lipids: metabolism. mutants. and membranes. Science. 252. 80-87.

Sträter.N.. Klabunde.T.. Tucker.P.. Witzel.H. and Krebs.B. (1995) Crystal structure of a purple acid phosphatase. Science. 268. 1489-1492. 


\section{Y.Lindquist et al.}

Strittmatter,P., Spatz,L., Corcoran,D., Rogers,M.J., Setlow,B. and Redline,R. (1974) Purification and properties of rat liver microsomal stearoyl coenzyme A desaturase. Proc. Natl Acad. Sci. USA, 71, 4565-4569.

Stukey,J.E., McDonough,V.M. and Martin,C.E. (1990) The OLE1 gene of Saccharomyces cerevisiae encodes the $\Delta 9$ fatty acid desaturase and can be functionally replaced by the rat stearoyl-CoA desaturase gene. J. Biol. Chem., 265, 20144-20149.

Thiede,M.A., Ozols,J. and Strittmatter,P. (1986) Construction and sequence of cDNA for rat liver stearoyl coenzyme A desaturase. J. Biol. Chem., 261, 13230-13235.

Thompson,G.A., Scherer,D.E., Foxall-Van Aken,S., Kenny,J.W., Young,H.L., Shintani,D.K., Kridl,J.C. and Knauf,V.C. (1991) Primary structure of the precursor and mature forms of stearoyl-acyl carrier protein desaturase from sunflower embryos and requirement of ferridoxin for enzyme activity. Proc. Natl Acad. Sci. USA, 88, $2578-2582$.

Received on March 19, 1996; revised on April 29, 1996 